\title{
ESTRATÉGIAS PARA TRABALHAR COM O LÚDICO DIANTE DO ISOLAMENTO SOCIAL POR CORONAVÍRUS: RELATO DE EXPERIÊNCIA ${ }^{1}$
}

\author{
STRATEGIES FOR WORKING WITH OF THE PLAY IN THE FACE OF \\ ISOLATION BY CORONAVIRUS: AN EXPERIENCE REPORT
}

\author{
Andreia Eckert Frank ${ }^{2}$, Luana Bartsch², Mariana Henrich Cazuni', \\ Tainara Giovana Chaves de $\operatorname{Vargas}^{2}$ e Andressa da Silveira ${ }^{3}$
}

\section{RESUMO}

A pandemia causada pelo novo coronavírus modificou o cotidiano da população, sobretudo das crianças e adolescentes que encontram-se restritas em seu domicílio. $\mathrm{O}$ isolamento social prolongado pode refletir no desenvolvimento infantil que passou por severas restrições. Desta forma, as atividades lúdicas como brincadeiras, jogos, dança, teatro podem ser estratégias benéficas para a promoção do cuidado e educação em saúde. Este artigo objetiva relatar a experiência de estudantes de enfermagem, integrantes do Projeto de Extensão "O lúdico e o brinquedo terapêutico como possibilidade para o cuidado de enfermagem" sobre atividades desenvolvidas a partir do uso de tecnologias como estratégia para o cuidado. Trata-se de um relato de experiência, sobre as vivências de estudantes de enfermagem na extensão, com ênfase em ações lúdicas, a partir das tecnologias digitais, que possibilitaram o contato virtual, diante da necessidade de isolamento. O uso da música, do teatro, de vídeos, desperta a imaginação e a curiosidade de crianças e adolescentes. Falar sobre cuidados de saúde utilizando esses recursos aproxima essa população dos profissionais, resultando em uma assistência humanizada e direcionada. Conclui-se que as atividades de cunho lúdico são importantes para o desenvolvimento infantil, inclusive, diante do caráter excepcional da pandemia. Sugere-se, o uso das tecnologias e das mídias sociais, visto que podem ser aliadas das práticas extensionistas de enfermagem.

Palavras-chave: Enfermagem pediátrica, Pandemias, Rede social, Tecnologia.

\section{ABSTRACT}

The pandemic caused by the new coronavirus has changed the daily lives of the population, especially children and adolescents who are restricted in their homes. Prolonged social isolation can reflect on child development that has undergone severe restrictions. Thus, recreational activities such as games, games, dance, theater can be beneficial strategies for the promotion of health care and education. This article aims to report the experience of nursing students, members of the Extension Project "Playful and therapeutic toys as a possibility for nursing care" on activities developed from the use of technologies as a possibility for care. It is an experience report, about the experiences of nursing students in extension, with emphasis on playful actions, based on digital technologies, which enabled virtual contact, in the face of the need for isolation. The use of music, theater, videos, awakens the imagination and curiosity of children and adolescents. Talking about health care

\footnotetext{
${ }^{1}$ Relato de experiência sobre o Projeto de Extensão "O lúdico e o brinquedo terapêutico como possibilidades para o cuidado de enfermagem".

${ }^{2}$ Acadêmicas do Curso de Graduação em Enfermagem, Universidade Federal de Santa Maria (UFSM), Campus de Palmeira das Missões. E-mails: andreiafrank93@gmail.com; luanabartsch2015@gmail.com; marianacazuni15@gmail.com; tainara.giovana.vargas73@gmail.com

${ }^{3}$ Professora Coordenadora do Projeto de Extensão. Doutora em Enfermagem. Docente do Departamento de Ciências da Saúde, Universidade Federal de Santa Maria (UFSM), Campus de Palmeira das Missões. E-mail: andressadasilveira@, gmail.com
} 
using these resources brings this population closer to professionals, resulting in humanized and targeted assistance. It is concluded that playful activities are important for child development, even in the face of the exceptional character of the pandemic. It is suggested, the use of technologies and social media, since they can be allied with extension nursing practices.

Keywords: Pediatric nursing, Pandemics, Social network, Technology.

\section{INTRODUÇÃO}

O coronavírus pertence a uma família de vírus que causa infecções respiratórias, sendo que os primeiros coronavírus humanos foram isolados em 1937. Já em 1965, o vírus foi descrito como coronavírus, em decorrência do perfil na microscopia, semelhante a uma coroa. O novo coronavírus ou SARS-CoV-2 foi descoberto ao final de 2019, na China, a doença denominada de COVID-19 apresenta um quadro clínico que varia entre infecções assintomáticas a quadros respiratórios graves (BRASIL, 2020a; ORGANIZAÇÃO PAN-AMERICANA DA SAÚDE, 2020). No Brasil, o primeiro caso foi registrado em fevereiro de 2020, no momento atual, a doença atinge o país de forma exponencial (BRASIL, 2020b).

Entre os principais sintomas da COVID-19 destacam-se: tosse, febre, coriza, dor de garganta e dificuldade para respirar. A doença é transmitida através do contato entre pessoas, por meio de gotículas de saliva, espirro ou tosse, contato das mãos com superfícies contaminadas que posteriormente podem encostar nos olhos, nariz ou boca. Entre as estratégias de prevenção sugere-se a lavagem das mãos, o uso de álcool gel $70^{\circ}$, o isolamento social voluntário, o uso de máscaras descartáveis ou de tecido, manter distância de dois metros entre as pessoas, ambientes limpos e ventilados (BRASIL, 2020; ORGANIZAÇÃO PAN-AMERICANA DA SAÚDE, 2020).

Pessoas com condições de saúde pré-existentes tendem a desenvolver formas mais graves da doença. A partir dos casos notificados, observa-se que a doença é relativamente rara e leve na população de crianças e adolescentes (ORGANIZAÇÃO PAN-AMERICANA DA SAÚDE, 2020; LIMA, 2020). Contudo, cabe aos pais ou responsáveis explicar para as crianças o que está ocorrendo, orientar a respeito das medidas de prevenção e tranquilizá-las, a fim de evitar os momentos de insegurança e ansiedade provocados pelo isolamento social (SOCIEDADE BRASILEIRA DE PEDIATRIA, 2020).

O "novo normal" e a necessidade de isolamento devido a pandemia de coronavírus, fez com que as pessoas mudassem suas rotinas. Com isso, as crianças encontram-se restritas no espaço domiciliar, a fim de reduzir a circulação do vírus. O isolamento social, pode ser vivenciado de diferentes maneiras. O nível de compreensão das crianças, a faixa etária, as circunstâncias sociais e os tipos de vulnerabilidade em que estão expostas também influenciam nas formas como o isolamento será vivenciado (FERRARI, 2020). 
O isolamento social prolongado pode prejudicar o desenvolvimento de crianças e adolescentes, visto que a socialização é fundamental nessas etapas da vida. Deste modo, o cotidiano permeado pelas relações sociais e familiares, com a consolidação das amizades e aquisição de novos conhecimentos, bem como brincadeiras, acaba restrito. Neste sentido, a utilização da ludicidade pode ser benéfica, e deve estar presente no cotidiano de crianças e adolescentes (SEIFER, 2020). Essas ações podem ser representadas através de brincadeiras, jogos, dança ou teatro. Elas possibilitam que crianças e adolescentes trabalhem suas potencialidades, por meio da imaginação (OLIVEIRA, 2016; VEIGA, 2016).

Frente a essas assertivas, acredita-se que as atividades lúdicas são potentes instrumentos para o desenvolvimento do vínculo, promoção do cuidado e para estabelecer práticas de educação em saúde com crianças e adolescentes no período de isolamento social. Este artigo objetiva relatar a experiência de estudantes de enfermagem, integrantes do Projeto de Extensão "O lúdico e o brinquedo terapêutico como possibilidades para o cuidado de enfermagem" sobre atividades lúdicas utilizando tecnologia como estratégia para o cuidado.

\section{MÉTODOS}

Trata-se de um estudo descritivo, na modalidade relato de experiência sobre as vivências de estudantes do curso de graduação em enfermagem no Projeto de Extensão "O lúdico e o brinquedo terapêutico como possibilidades para o cuidado de enfermagem" e as atividades lúdicas utilizando tecnologia e mídia social como estratégias para o cuidado.

Entre as ações de extensão, podem ser destacadas: educação em saúde com crianças e adolescentes hospitalizados, atividades lúdicas com crianças e adolescentes estudantes e/ou usuários da clínica da Associação de Pais e Amigos dos Excepcionais (APAE). A respeito das atividades desenvolvidas, salienta-se o livro lúdico, com contos que tratam sobre a promoção da saúde e prevenção de doenças, teatro lúdico e a desconstrução dos contos de fadas.

O Projeto de Extensão foi aprovado em 2018, e está em andamento até os dias atuais, por meio de estratégias virtuais que incorporam ações lúdicas e também relacionadas ao brinquedo terapêutico. As atividades possuem dois cenários: APAE e a Unidade Pediátrica de um Hospital de médio porte.

A partir de 18 de março de 2020, com a pandemia relacionada ao novo coronavírus tomando proporções exponenciais no país, as atividades presenciais foram suspensas. A docente e as estudantes passaram a utilizar as tecnologias e as mídias sociais, como estratégia de vínculo, cuidado e ludicidade com crianças e adolescentes.

A docente criou um Canal no YouTube denominado "Viver Enfermagem", com vídeos quinzenais, utilizando o espaço para a comunicação com estudantes, crianças, adolescentes e profissionais da saúde em prol das práticas de educação em saúde no espaço virtual. Com o uso de fantasias, máscaras, maquiagem e músicas infantis, os vídeos do projeto tornaram-se mais atrativos, utilizando 
a ludicidade no espaço virtual. Ao que se refere as orientações desenvolvidas nos vídeos, este relato apresenta resultados de três vídeos, com ênfase nos cuidados de saúde para prevenção de COVID-19.

\section{RESULTADOS}

O Projeto de Extensão passou a realizar encontros virtuais, utilizando uma Plataforma Google Meet que contemplou as possibilidades de desenvolver reuniões online entre estudantes e docente. Após três encontros virtuais foi sugerido o desafio de desenvolver as ações extensionistas utilizando as redes sociais. Desta forma, seria possível manter o vínculo e o contato com os cenários das ações, trazendo a educação em saúde e a informação para crianças e adolescentes.

As atividades foram definidas de forma coletiva em reuniões online. Os temas e o roteiro de gravação foi estabelecido pela docente, com auxílio das estudantes que participaram das gravações em suas residências. O material foi editado pela docente, disponibilizado no Canal e divulgado para os cenários do projeto.

O primeiro vídeo apresenta o motivo pelo qual as ações não estavam sendo realizadas de modo presencial. O vídeo construído intercalava a imagem das integrantes do projeto caracterizadas, dançando e cantando a música "Cabeça, ombro, joelho e pé”. Ele abrange a perspectiva do projeto em si, o lúdico e as brincadeiras como potencial aliado ao cuidado à saúde e sobretudo, a respeito de promover a descontração, mesmo diante da pandemia.

O segundo vídeo salienta a importância do uso de máscaras ao sair de casa. Utilizou-se a música "Carimbador maluco" e é dividido em dois momentos: no primeiro bloco observa-se as estudantes e docente sem máscara, fazendo sinais de negação ao trecho de "não vai a lugar nenhum". Já o segundo bloco é sinalizado de forma positiva, no momento em que é apresentado o trecho "pode sair sem problema algum”, enfatizando sobre o uso de máscaras. Esse vídeo evidenciou sobre um momento particular vivido em 2020, onde crianças e adolescentes precisaram se adaptar a uma nova realidade, promovendo orientações sobre cuidado e empatia.

O terceiro vídeo expõe ações do cotidiano, as quais necessitaram ser modificadas devido à pandemia causada pelo SARS-CoV-2. O vídeo apresentou estratégias preventivas, utilizando a metáfora do lúdico e do "certo ou errado" como um quis para crianças e adolescentes. Foi abordado sobre higiene, lavagem de mãos, uso de álcool gel, utilização de máscaras e inclusive a forma de tossir e espirrar em ambientes públicos.

As crianças e adolescentes trouxeram um feedback positivo, o que também revelou que a enfermagem pode e deve utilizar as diversas formas de tecnologias e redes sociais, para fazer práticas de educação em saúde. Os vídeos produzidos foram bem aceitos pelos cenários onde o projeto tem sido realizado. A partir dessas produções, outros estudantes de enfermagem procuraram o projeto, com intuito de serem voluntários e de construírem novas proposições. 
Estes resultados evidenciam a necessidade da extensão ser trabalhada de forma acessível e com intuito de promover atividades para crianças e adolescentes, a partir de suas vivências. Com a pandemia e a necessidade de isolamento, o uso de tecnologias e das redes sociais são possibilidades para o cuidado de enfermagem.

\section{DISCUSSÃO}

O uso do lúdico pode beneficiar as crianças e adolescentes, visto que a utilização da imaginação e das habilidades tornam a educação em saúde acessível. Os dispositivos tecnológicos somados ao lúdico, aproxima a criança do profissional, resultando em uma assistência humanizada. Em tempos de pandemia, o uso de tecnologias leves podem ser possibilidades para as relações afetivas e de vínculo (SANTOS, 2019).

Estudos indicam que a utilização de redes sociais nos processos de trabalho de enfermagem é recente, porém estão crescendo a cada ano e atingindo um público com diferentes perfis e, devido a facilidade de acesso, podem alcançar diversas populações (MESQUITA, 2017). Isso foi percebido com a divulgação dos vídeos, que podem ser assistidos no Canal do YouTube e compartilhados por meio das redes sociais, atingindo pessoas de diferentes idades, sexos e classes sociais.

Entretanto, o acesso às redes sociais e mídias digitais não ocorre de forma igualitária para todas as crianças e adolescentes. Isso pode atingir, principalmente, àquelas socialmente vulneráveis, devido suas precárias condições de acesso educacional. Ademais, percebe-se que os momentos de crise podem ser duradouros, refletindo em falta de proteção, risco para violência e abuso, expondo-as ainda mais, aos diversos tipos de carência e vulnerabilidades (THE LANCET PUBLIC HEALTH, 2020).

Diante da situação de isolamento social imposta pela pandemia, os parâmetros para a construção do aprendizado foram reinventados, sendo incorporado o uso das tecnologias para continuidade do ensino (KOBAYASHI; LEITE, 2015). Cada vez mais cedo, crianças e adolescentes utilizam tecnologias, seja na esfera do entretenimento, aprendizado ou comunicação. A partir do uso das mídias digitais e de dispositivos tecnológicos, crianças e adolescentes criam seus próprios espaços de autonomia (MONTEIRO, 2015).

Os recursos tecnológicos podem ser utilizados para fins de educação em saúde e pesquisa, além de serem aliados no processo ensino-aprendizagem para alunos da graduação, contribuindo como uma nova forma de produzir conhecimento e abertura a novos métodos de ensino (MESQUITA, 2017; BEZERRA, 2020). Para as estudantes participantes do projeto, foi possibilitada uma aproximação, que por hora não era possível presencialmente, mas que, virtualmente contribuiu na formação profissional, permitindo aprender novas maneiras de fazer extensão junto à comunidade.

O lúdico tem um papel muito importante, pois permite estimular as crianças e adolescentes a desenvolverem suas habilidades, utilizar a imaginação, estabelecer vínculo e comunicação a partir 
de sua compreensão. Ademais, as ações lúdicas despertam o interesse e curiosidade. Esses fatores trazem benefícios, inclusive para o desenvolvimento cognitivo, bem como nos aspectos físicos, emocionais, afetivos, culturais e sociais. As atividades diversificadas podem desenvolver habilidades motoras, reduzir a agressividade, exercitar a criatividade, além de aprimorar a inteligência emocional e adaptação social (SILVA, 2020).

A utilização de tecnologias podem ser benéficas, quando associadas a temas que ressaltam sobre as diferenças, a valorização das pessoas, a educação e a sensibilidade humana. O uso de tecnologias deve ser capaz de incluir crianças e adolescentes, a fim de que professores e estudantes percebam o seu efeito no desenvolvimento humano, e na apropriação dos conhecimentos, como possibilidade para a transformação da realidade (CONTE; OURIQUE; BASEGIO, 2017). As tecnologias digitais podem operar como recursos de empoderamento, ampliando a integração social, por meio de atividades digitais, vídeos, jogos e interação que melhoram as condições cognitivas, afetivas e emocionais (SANTAROSA; CONFORTO, 2015).

As crianças com necessidades educacionais especiais, como aquelas com transtorno do espectro autista, também se encontram em risco no período de isolamento. Elas podem ficar frustradas e irritadiças quando suas rotinas diárias são interrompidas. Isso enaltece a necessidade dos pais ou responsáveis desenvolverem um cronograma para essas crianças, além de formas criativas para ensinar e brincar por meio de atividades lúdicas, com intuito de reduzir a ansiedade que é causada pela incerteza do momento atual (LEE, 2020). Dessa forma, é essencial refletir sobre a acessibilidade e o potencial das tecnologias para o público de crianças e adolescentes, onde o uso do lúdico e das mídias sociais são alternativas para a promoção do cuidado em saúde.

\section{CONSIDERAÇÕES FINAIS}

Este artigo proporcionou relatar a experiência de estudantes de enfermagem sobre ações de extensão desenvolvidas diante do isolamento social atrelado a pandemia de COVID-19. Os parágrafos construídos e as leituras realizadas, resgatam sobre a importância das atividades lúdicas para crianças e adolescentes, visto que a aquisição de novos conhecimentos e o desenvolvimento são constantes.

Para as crianças, as atividades lúdicas somadas ao ato de brincar são essenciais para o uso da imaginação, segurança e também, como um elo de comunicação. Já para os adolescentes, a utilização dos espaços virtuais aproximou ainda mais, a enfermagem desse público, além de haver maior interação e participação.

Apesar de as ferramentas virtuais terem sido pensadas como possibilidades para o cuidado e educação em saúde frente a necessidade de isolamento social, observa-se que essas atividades podem estar associadas às ações presenciais. Os vídeos produzidos pelo Canal Viver Enfermagem 
alcançaram públicos maiores, houve adesão de crianças e adolescentes, difundindo informação de forma lúdica, por meio das práticas de educação em saúde. Esses achados são positivos e benéficos para a construção de saberes entre a universidade e comunidade, além de fortalecerem o papel social da extensão.

O uso da dramatização, os roteiros construídos, a música e os vídeos tornam o cuidado de enfermagem mais acessível e sensível, as reais demandas de crianças e adolescentes. Por fim, recomenda-se a utilização das redes sociais e das tecnologias, aliadas às práticas de enfermagem, inclusive em situações de caráter excepcional, como as de isolamento social por coronavírus.

\section{REFERÊNCIAS}

BEZERRA, I.M.P. Estado da arte sobre o ensino de enfermagem e os desafios do uso de tecnologias remotas em época de pandemia do coronavírus. Journal of Human Growth and Development, v. 30, n. 1, p. 141-147, 2020. Disponível em: https://bit.ly/3np6uY3. Acesso em: 01 jun. 2020.

BRASIL. Ministério da Saúde. O que é COVID-19. Disponível em: https://bit.ly/35mbvu2. Acesso: 01 jun. 2020-a.

BRASIL. Ministério da Saúde. Painel Coronavírus. Disponível em: https://covid.saude.gov.br/. Acesso em: 01 jun. 2020-b.

Organização Pan-Americana da saúde. Folha informativa COVID-19 - Escritório da OPAS e da OMS no Brasil. Disponível em: https://www.paho.org/pt/covid19. Acesso em: 01 jun. 2020.

CONTE, E.; OURIQUE, M.L.H.; BASEGIO, A.C. Tecnologia assistiva, direitos humanos e educação inclusiva: uma nova sensibilidade. Educação em revista, v. 33, e163600, 2017.

FERRARI, A.; CUNHA, A.M. A pandemia do Covid-19 e o isolamento social: saúde versus economia. Faculdade de Ciências Econômicas da UFRGS, 2020. Disponível em: https://bit.ly/2JRKRBN. Acesso em: 01 jun. 2020.

KOBAYASHI, R.M.; LEITE, M.M.J. Competências tecnológicas no ensino de enfermagem cardiovascular. Revista da Escola de Enfermagem da USP, v. 49, n. 6, p. 971-977, dez. 2015. Disponível em: https://bit.ly/2XmbCkV. Acesso em: 01 jun. 2020. 
LEE, J. Mental health effects of school closures during COVID-19. The Lancet Child \& Adolescent Health, v. 4, n. 6, p. 421, 2020. Disponível em: https://bit.ly/3hQBYVL. Acesso em: 01 jun. 2020.

LIMA, C.M.A.O. Informações sobre o novo coronavírus (COVID-19). Radiologia Brasileira, v. 53, n. 2, p. V-VI, abr. 2020. Disponível em: https://bit.ly/3bj3g5K. Acesso em: 01 jun. 2020.

MESQUITA, A.C. et al. As redes sociais nos processos de trabalho em enfermagem: revisão integrativa da literatura. Revista da Escola de Enfermagem da USP, v. 51, e03219, mar. 2017. Disponível em: https://bit.ly/3s06Zey. Acesso em: 01 jun. 2020.

MONTEIRO, A.F.; OSÓRIO, A.J. Novas tecnologias, riscos e oportunidades na perspectiva das crianças. Revista Portuguesa de Educação, v. 28, n. 1, p. 35-57, jun. 2015. Disponível em: https://bit.ly/ 3nnBBTN. Acesso em: 01 jun. 2020.

OLIVEIRA, S.R.; ALMEIDA, A.N. As expressões lúdicas como terapêutica na hospitalização: revisão integrativa. Revista Eletrônica Gestão \& Saúde, v. 7, n. 1, p. 356-68, 2016. Disponível em: https://www.periodicos.unb.br/index.php/rgs/index/. Acesso em: 01 jun. 2020.

SANTAROSA, L.M.C.; CONFORTO, D. Tecnologias móveis na inclusão escolar e digital de estudantes com transtornos de espectro autista. Revista Brasileira de Educação Especial, v. 21, n. 4, p. 349-366, dez. 2015. Disponível em: https://bit.ly/38oC3Nj. Acesso em: 01 jun. 2020.

SANTOS, J. M. S.; SILVA, M. E. B.; ARAÚJO, R. J. S.; LOPES, R. F.; CALDAS, M. A. G. Atividades lúdicas e educação em saúde com crianças hospitalizadas: um relato de experiência projeto resgatar. GEPNEWS, v. 2, n. 2, p. 616-623, jun. 2019. Disponível em: https://bit.ly/3biHSOb. Acesso em: 01 jun. 2020.

SEIFER, K. Rotina e conversa franca auxiliam crianças a enfrentar isolamento. O diário de Mogi das Cruzes, 2020. Disponível em: https://odiariodemogi.net.br/. Acesso em: 01 jun. 2020.

SILVA, K. F. da. O Lúdico na Educação Infantil: limites e possibilidades. Trabalho de Conclusão de Curso. Instituto Federal de Ciência e Tecnologia Goiano - Campus Morrinhos, 2020, 58p. Disponível em: https://bit.ly/398yk5z. Acesso em: 01 jun. 2020.

\section{SOCIEDADE BRASILEIRA DE PEDIATRIA. Orientações a Respeito da Infecção pelo SARS-CoV-2} (conhecida como COVID-19) em Crianças. Departamento Científico de Infectologia, mar. 2020. Disponível em: https://bit.ly/3bf8kIw. Acesso em: 01 jul. 2020. 
THE LANCET PUBLIC HEALTH. COVID-19 puts societies to the test. The Lancet. Public health, v. 5, n. 5, e235, 2020. Disponível em: https://bit.ly/3s3vuY1. Acesso em: 01 jun. 2020.

VEIGA, M.A.B.; SOUSA, M.C.; PEREIRA, R.S. Enfermagem e o brinquedo terapêutico: vantagens do uso e dificuldades. Revista Atualiza Saúde, v. 3, n. 3, p. 60-66, 2016. Disponível em: https://bit.ly/ 2La5dHa. Acesso em: 01 jun. 2020. 
